\title{
Operative Management of Type II Endoleaks After Aortic Endovascular Repair
}

\author{
Anna Maria lerardi, Filippo Pesapane, \\ Francesca Patella, Enrico Maria Fumarola, \\ Salvatore Alessio Angileri, Mario Petrillo, \\ Matteo Crippa, and Gianpaolo Carrafiello
}

\subsection{Introduction}

Endoleaks (ELs) are the most common complications after endovascular aneurysm repair (EVAR), with an incidence that varies between $10 \%$ and $50 \%$ [1-4]. They are defined as persistent flow within the excluded aneurysm sac and the graft [1-4]. Endoleaks are classified according to the underlying etiology and localization $[5,6]$. The risk of rupture of the aneurysmatic sac depends on the type and persistency of endoleaks. The type II endoleak (T2EL) is the most common form and occurs in about $20-30 \%$ of patients after treatment, persisting in $10-15 \%$ of patients after 6 months [3]. T2EL occurs when a retrograde flow of blood supplies the aneurysm sac through branch vessels such as lumbar arteries or a patent inferior mesenteric artery (IMA) [4]. T2EL usually has a complex architecture because it should be featured by more than one inflow and

\footnotetext{
A. M. Ierardi · S. A. Angileri · M. Petrillo G. Carrafiello $(\bowtie)$ Department of Health Sciences, Diagnostic and Interventional Radiology, San Paolo Hospital, University of Milan, Milan, Italy e-mail: gianpaolo.carrafiello@unimi.it

F. Pesapane - F. Patella - E. M. Fumarola Postgraduation School in Radiodiagnostics, Università degli Studi di Milano, Milan, Italy

M. Crippa

Surgery Department, San Paolo Hospital, University of Milan, Milan, Italy
}

outflow vessel, which communicates through a channel [5]. For this reason, a further differentiation of T2EL was proposed: type IIA, when it is related to only one patent branch, and type IIB, when it is complex with two or more patent branches and creating a flow-through situation [4].

Fifty percent of type II ELs resolve spontaneously, $10-15 \%$ are persistent on long-term follow-up, and recurrent T2EL develop in $5-10 \%$ $[2,4,7,8]$.

A conservative approach with regular followup is accepted for stable T2EL [9, 10], whereas treatment of T2EL is normally required when the aneurysm sac diameter increases more than $5 \mathrm{~mm}$ between sequential Computed Tomography (CT) follow-up examinations, because this is indicative of high sac pressure [3].

In the past, T2EL were usually treated surgically, both with graft explantation and with retroperitoneal ligation of collateral feeding vessels [5]. These invasive methods require hospital admission and they increase morbidity of surgery [11]. For this reason, over the years, new less invasive techniques for the treatment of T2EL have made their way through. Nowadays, the most common treatment for patients with T2EL is transarterial embolization (TAE), with the aim of occluding the feeding arteries and/or the EL nidus [12]. In order to achieve successful embolization, the inflow vessels and the channel(s) need to be embolized [5]. A critical step of the procedure is 
the identification of the artery feeding the sac, which allows reaching the nidus of the endoleak and delivering embolic agents for complete occlusion. Failure is primarily due to the inability to reach the nidus or to coil the aneurysmatic sac [13].

Angiographic identification of the feeding artery may be time-consuming, because sequential angiographic image acquisitions are usually necessary. Moreover, determination of the specific artery responsible for the EL is difficult when the arterial vasculature has a complex branching pattern [13, 14]. Several studies presented the value of intraprocedural Cone-Beam CT (CBCT) and Advanced Vessel Detection (AVD) software as problem-solving tool to aid in safe catheterization of offending vessels during TAE of gastrointestinal $[15,16]$ and visceral arterial bleedings $[4,17,18]$ while decreasing total procedure time, contrast dose, and radiation exposure $[15,19]$; recently the same technology was used to detect the feeders of the ELs $[15,16]$. When TAE is not feasible (e.g., there are no arterial routes) or unsuccessful, direct percutaneous sac injection (DPSI) and transcaval embolization (TCE) are alternative endovascular methods validated for the treatment of T2EL [5, 20-23].

Actually, no treatment is considered the gold standard for T2EL management, and consequently, a tailored treatment should be preferred $[1,23,24]$.

A variety of embolic agents have been used either in isolation or in combination for treatment of T2EL [5]. Liquid embolic agents and coils are the main embolic agents used [12], but there are no guidelines for the choice of embolic material, and the final decision is often made on the basis of the operator experience and confidence [5].

Patient preparation and management is the same as for any standard endovascular procedure. Prophylactic antibiotics for secondary interventions are advisable, although there is no convincing evidence of benefit for the prevention of infection in the literature [4].

\subsection{Endovascular Embolization}

Percutaneous TAE is the most common intervention for T2EL [12]. This procedure involves a percutaneous transfemoral or transbrachial approach, with the aim of catheterizing the nidus of the endoleak via its collaterals.

Treatment of T2EL is feasible when IMA is supplied via the arch of Riolano through the collaterals of the SMA. Another possible approach is from the internal iliac arteries when the endoleak arises from lumbar arteries (LA), through collaterals of the iliolumbar arteries [25].

Briefly, for transarterial embolization of an IMA T2EL, catheterization of the middle colic artery (MCA) through the SMA, a microcatheter must be advanced via the arch of Riolano until the catheterization of the IMA [26] (Fig. 9.1). With regard to the embolization of a LA T2EL, a short sheath is
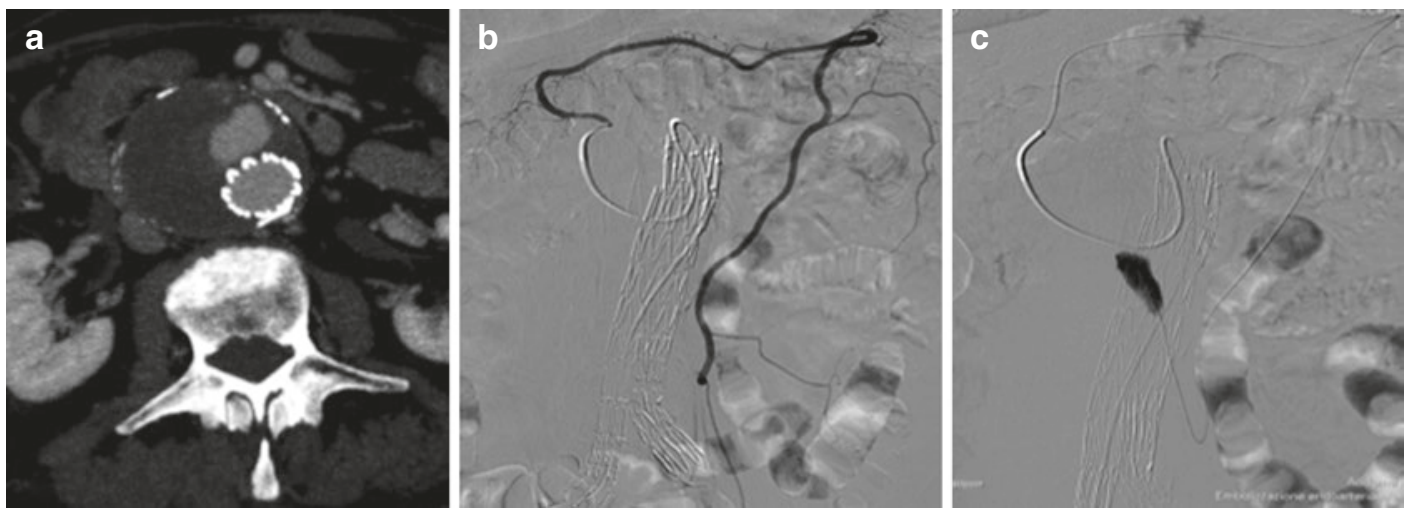

Fig. 9.1 CT Angiography revealed T2EL supplied from IMA (a); a microcatheter has to be advanced via the arch of Riolano until the catheterization of the IMA (b) and to reach the nidus (c) 

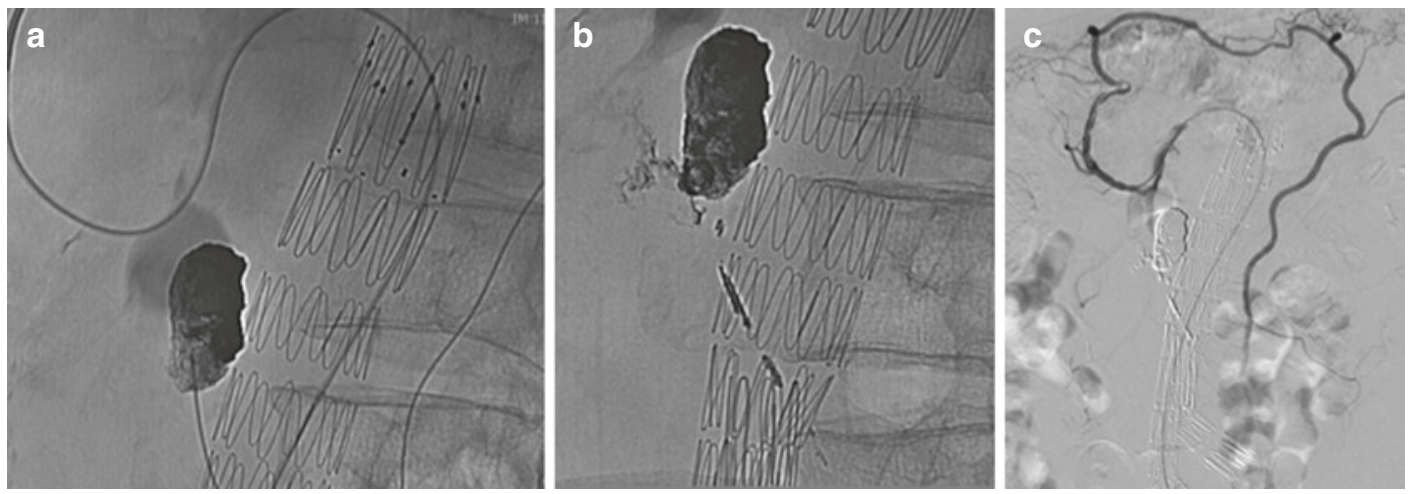

Fig. 9.2 Endoleak nidus was reached and the embolic agent was injected to fill the nidus and the feeding vessel (a, b); final angiogram confirmed complete embolization of the EL (c)

placed into the ipsilateral femoral artery, and the internal iliac artery is catheterized. As the majority of lumbar T2EL communicate with the iliolumbar artery, it is generally necessary to catheterize the iliolumbar artery with a microcatheter and an angled microwire, a passage which can be extremely challenging [26].

In both cases, when the EL's nidus is reached, it is possible to inject or deploy the embolic agent until the nidus and the feeding vessels are occluded [12] (Fig. 9.2).

This procedure may be difficult, timeconsuming, and not possible in all patients because of anatomic limitations [27]; as literature data has demonstrated, embolization of a single feeding or draining artery may be ineffective in most cases, because additional flow is often recruited from other adjacent branches [14]; it is highly suggested to try to achieve a permanent cessation of flow occluding the entire nidus, in order to interrupt the communication to all patent communicating aortic side branches, including the inflow and outflow vessels [28].

The final goal is to definitively stop the blood flow in these collateral arteries with appropriate embolic agents which should cause thrombosis and cessation of the EL [29].

EL embolization can be performed with many different embolic agents. Glue (n-butyl cyanoacrylate (NBCA)), coils, thrombin, and polymers (ethylene-vinyl alcohol copolymer $(\mathrm{EVOH})$ ) are the most commonly used ones [30].
Among these agents, polymer Onyx (Liquid Embolic System; ev3 Neurovascular, Irvine, California) is particularly suitable for EL embolization. $\mathrm{EVOH}$ is dissolved in dimethyl sulfoxide (DMSO). When Onyx comes into contact with blood, it precipitates and forms a cast, with a harder outer layer and a semiliquid central core. These proprieties can be helpful especially in cases with tortuous and small feeding vessels, when microcatheter positioning into the nidus is unachievable. In these situations, Onyx can be injected from a more distant catheter position through the network of collateral vessels and pushed into the nidus, thanks to its unique proprieties [31].

However, a recently-published retrospective study, despite an encouraging post-procedural high success rate $(88.9 \%)$, after a mean follow-up period of 32.8 months, $44.4 \%$ of patients required a second intervention for EL recurrence or delayed clinical failure [32].

So, despite some encouraging results on Onyx and other liquid agents, there are no conclusive data on the advantages or disadvantages of the different embolic materials [26].

A large and recent review that considered seven different studies which reported outcomes for transarterial procedures, for a total of 120 interventions, showed an overall clinical success rate of $62.5 \%$, defined as no evidence of recurrent T2EL during follow-up. In this cohort of patients, $22.5 \%$ required further intervention for failed embolization 
[33]: development of new type II ELs, persistent blood flow through the coils of the treated EL, and growth of anastomoses around the coiled vessel are all reasons suggested as being correlated to clinical failure [2].

As already pointed out, the critical step in the procedure is to identify the feeding sac artery, which then allows to reach the nidus and deliver embolic agents for complete occlusion. Failure occurs primarily due to an inability to reach the nidus or to embolize the aneurysmatic sac [13].

Technology is moving fast and new tools are available and can help to spare time and to improve outcomes. In particular, C-arm CBCT is considered the most impactful breakthrough in the last decade [34].

CBCT and AVD software may be a useful option in case of angiographically occult T2EL or complex vasculature, permitting to identify safely and quickly the feeding artery [15].

\subsection{Percutaneous Sac Puncture}

Percutaneous sac puncture (PSP) is an interventional procedure consisting in the direct injection of an embolizing agent into the aneurysmatic sac through a percutaneous access [20].

It has been proposed as an alternative option for treatment of T2EL in case of unsuccessful TAE $[4,35]$ or whether endovascular approach is not feasible [20].

PSP can be performed with two different techniques: with a transabdominal or a translumbar approach (Fig. 9.3). The first method should be preferred if the EL is located or extended anteriorly, while the second one should be selected whether the EL is visualized in the posterior portion of the sac or when bowel or other important structures are interposed [25, 36]. Indeed, the most important advantage of the translumbar approach is that it does not imply the passage into cavities or organs [35, 37]. However, this method imposes an uncomfortable position of the patient, who is supposed to stay in a prone position [36].

Conversely, the principal advantage of the transabdominal technique is that the patient can be placed in a supine position; moreover a simultaneous transarterial angiography should be performed if needed. The risk of organ injury during the transabdominal approach can be minimized, using real-time US guidance [36].

In both cases, in order to confirm the T2EL and to assess its origin (lumbar and/or mesenteric), PSP requires a pre-interventional imaging evaluation, that can be provided either by CEUS [5] or CT angiography or MRI angiography [36] or CT/MRI and CEUS.

The procedure can be performed under local anesthesia [20].

Briefly, PSP technique involves the percutaneous puncture under US or CT/fluoroscopic guidance of the aneurysmatic sac, generally using a $21 \mathrm{G}$ needle [36]. Once blood flashback is obtained, a wire is advanced into the sac, to allow the positioning of a larger introducer sheath and the subsequent introduction of a 4 or $5 \mathrm{~F}$ angiographic catheter [25]. Then, after an angiogram of the aneurysmatic sac to assess the features of the EL and to identify the inflow and
Fig. 9.3 PSP can be performed with translumbar (a) or transabdominal (b) approach
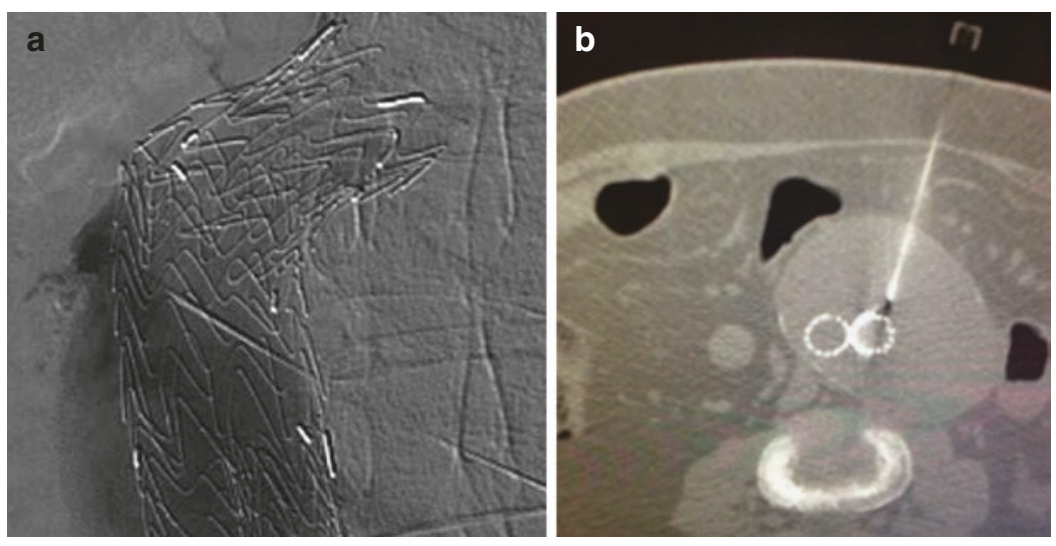
outflow vessels, the embolization of the sac and of the feeding vessels can be performed $[25,36]$.

As above reported, there are no guidelines for the choice of the embolic material; a variety of embolic agents either combined or alone can be used for the treatment of T2EL. However, permanent agents such as coils, NBCA, thrombin, and EVOH are advisable [5]. In addition, the selection of a liquid embolic agent allows to completely fulfilling the aneurysmatic sac, including all in- and outflow vessels, creating a solid cast which results in an uncompressible structure that impedes recanalization, providing a hypothetic more durable repair than coils. Specifically, EVOH has a lava-like flow pattern within blood vessels without any fragmentation during the injection. It is also associated with a lower inflammatory reaction and the absence of polymerization heat compared with NBCA [38].

Featured by almost $100 \%$ of technical success rate and $70 \%$ of secondary clinical success rate, percutaneous and endovascular treatments have overlapping outcomes [25], but PSP is usually a faster procedure, able to reduce consistently the ionizing radiation dose, especially if US-guidance is used.

Complications are rare and include nontarget embolization, puncture site hematomas, and perforation of vessels or bowel during the passage of the needle [25].

However, treatment results must be checked by a strict imaging follow-up, generally provided by CT angiography or Contrast-Enhanced UltraSound (CEUS) at 30 days, at 6 months, and at 12 months postoperatively and annually thereafter [5].

In conclusion, PSP can be a helpful minimally invasive therapeutic option for treatment of T2EL, allowing the sac obliteration, as well as a significantly shorter procedure and fluoroscopy time compared to endovascular approach.

\subsection{Transcaval Embolization}

TCE has been proposed instead of the direct translumbar approach as the initial treatment of T2EL and in cases where transarterial embolization failed. The indications for treatment are the same as those reported in the introduction of this chapter [24].
Compared with TAE, TCE is less timeconsuming and can be also performed in T2EL with no direct lumbar collateral vessels. Another advantage of TCE is the utilization of supine positioning, which facilitates patient comfort and concomitant arterial access allowing the confirmation of the EL, verification of EL obliteration and, if necessary, concurrent TAE. If the T2EL originates from lumbar or middle sacral vessel, we directly access the aorta via either a translumbar or TCE, with the approach dictated by the localization of the T2EL and the aorta relative to the inferior vena cava (IVC) and lumbar spine [39]. Furthermore, with TCE, the IVC is punctured through one wall instead of both, as required when the right translumbar approach is performed, and lower risk of retroperitoneal hemorrhage or inadvertent bowel injury may be anticipated [24, 39].

The TCE is viable in almost all patients; however, direct puncture should be difficult when the aneurysmatic sac diameter is small and is associated with an increased risk of stent graft puncture.

Preoperative planning allows identification of radiographic markers (e.g., stent graft, graft bifurcation, device overlap points, or aortic wall calcifications) that may guide transcaval puncture facilitating the access to the T2EL [39]. In order to optimize needle orientation into the aneurysm sac, an intraoperative CT or CBCT can be performed $[15,19]$. After confirmation of successful embolization, the puncture system may be removed, and an imaging examination should be performed to determine if there is any evidence of aortocaval fistula or contrast extravasation. Usually the patient is admitted overnight and undergoes noncontrast CT at $24 \mathrm{~h}$ to evaluate the immediate success of embolization and to identify any complications related to the procedure [24, 39]. Potential complications include the thrombophlebitis at the site of femoral percutaneous venous access, inadvertent intracaval embolization of coils, retroperitoneal hematoma, and caval-aortic fistulas [24, 39]. However, TCE requires a thin needle cannula and a $5 \mathrm{~F}$ catheter, which is quite small in caliber and only causes small holes in the caval walls. The 
caval hole is expected to close immediately after the removal of the $5 \mathrm{~F}$ catheter $[21,24]$.

To the best of our knowledge, the cumulative combined reported success of transarterial and translumbar procedures is $\leq 70 \%$, and this is comparable to the early results of TCE [39].

Follow-up CT should be performed following the same protocol described above [24].

In conclusion, TCE seems to be a safe and technically feasible management option for T2EL which require treatment; nevertheless, further studies are needed to confirm its role as a complementary technique to existing methods of T2EL management.

\subsection{Conclusions}

The described techniques permit to treat T2EL and to avoid surgery, with the main advantages of mini-invasivity and less morbidity and mortality.

More studies with longer follow-up will allow for more information about embolic agents and tools to reach the nidus (CBCT and AVD software).

Compliance with Ethical Standards

Conflict of interest: The authors declare that they have no conflict of interest.

\section{References}

1. Veith FJ, Baum RA, Ohki T, et al. Nature and significance of endoleaks and endotension: summary of opinions expressed at an international conference. J Vasc Surg. 2002;35(5):1029-35.

2. Solis MM, Ayerdi J, Babcock GA, et al. Mechanism of failure in the treatment of type II endoleak with percutaneous coil embolization. J Vasc Surg. 2002;36(3):485-91.

3. Bryce Y, Rogoff P, Romanelli D, Reichle R. Endovascular repair of abdominal aortic aneurysms: vascular anatomy, device selection, procedure, and procedure-specific complications. Radiographics. 2015;35(2):593-615.

4. Rand T, Uberoi R, Cil B, Munneke G, Tsetis D. Quality improvement guidelines for imaging detection and treatment of endoleaks following endovascular aneurysm repair (EVAR). Cardiovasc Intervent Radiol. 2013;36(1):35-45.

5. Ierardi AM, Micieli C, Angileri SA, et al. Ethylenevinyl alcohol copolymer as embolic agent for treat- ment of type II endoleak: our experience. Radiol Med. 2017;122(2):154-9.

6. Gelfand DV, White GH, Wilson SE. Clinical significance of type II endoleak after endovascular repair of abdominal aortic aneurysm. Ann Vasc Surg. 2006;20(1):69-74.

7. Harris PL, Dimitri S. Predicting failure of endovascular aneurysm repair. Eur J Vasc Endovasc Surg. 1999;17(1):1-2.

8. Baum RA, Carpenter JP, Golden MA, et al. Treatment of type 2 endoleaks after endovascular repair of abdominal aortic aneurysms: comparison of transarterial and translumbar techniques. J Vasc Surg. 2002;35(1):23-9.

9. Moll FL, Powell JT, Fraedrich G, et al. Management of abdominal aortic aneurysms clinical practice guidelines of the European society for vascular surgery. Eur J Vasc Endovasc Surg. 2011;41(Suppl 1):S1-S58.

10. EVAR Trial Participants. Endovascular aneurysm repair and outcome in patients unfit for open repair of abdominal aortic aneurysm (EVAR trial2): randomised controlled trial. Lancet. 2005;365(9478):2187-92.

11. Wisselink W, Cuesta MA, Berends FJ, van den Berg FG, Rauwerda JA. Retroperitoneal endoscopic ligation of lumbar and inferior mesenteric arteries as a treatment of persistent endoleak after endoluminal aortic aneurysm repair. J Vasc Surg. 2000;31(6):1240-4.

12. Avgerinos ED, Chaer RA, Makaroun MS. Type II endoleaks. J Vasc Surg. 2014;60(5):1386-91.

13. Kasirajan K, Matteson B, Marek JM, Langsfeld M. Technique and results of transfemoral superselective coil embolization of type II lumbar endoleak. J Vasc Surg. 2003;38(1):61-6.

14. Nevala T, Biancari F, Manninen H, et al. Type II endoleak after endovascular repair of abdominal aortic aneurysm: effectiveness of embolization. Cardiovasc Intervent Radiol. 2010;33(2):278-84.

15. Ierardi AM, Pesapane F, Rivolta N, et al. Type 2 endoleaks in endovascular aortic repair: cone beam CT and automatic vessel detection to guide the embolization. Acta Radiol. 2018;59(6):681-7.

16. Patil VV, Fischman AM, Patel RS, et al. GI hemorrhage arising from splenic artery: intraprocedure cone-beam CT as problem-solving tool to aide in safe catheterization of offending vessel. Clin Imaging. 2015;39(5):928-30.

17. Miyayama S, Yamashiro M, Okuda $M$, et al. Usefulness of cone-beam computed tomography during ultraselective transcatheter arterial chemoembolization for small hepatocellular carcinomas that cannot be demonstrated on angiography. Cardiovasc Intervent Radiol. 2009;32(2):255-64.

18. Ronot M, Abdel-Rehim M, Hakime A, et al. Cone-beam CT angiography for determination of tumor-feeding vessels during chemoembolization of liver Tumors: comparison of conventional and dedicated-software analysis. J Vasc Interv Radiol. 2016;27(1):32-8.

19. Carrafiello G, Ierardi AM, Duka E, et al. Usefulness of cone-beam computed tomography and automatic 
vessel detection software in emergency transarterial embolization. Cardiovasc Intervent Radiol. 2016;39(4):530-7.

20. Park YS, Do YS, Park HS, Park KB, Kim DI. Experience of direct percutaneous sac injection in type II endoleak using cone beam computed tomography. Ann Surg Treat Res. 2015;88(4):232-5.

21. Stavropoulos SW, Carpenter JP, Fairman RM, Golden MA, Baum RA. Inferior vena cava traversal for translumbar endoleak embolization after endovascular abdominal aortic aneurysm repair. J Vasc Interv Radiol. 2003;14(9 Pt 1):1191-4.

22. Ellis PK, Kennedy PT, Collins AJ, Blair PH. The use of direct thrombin injection to treat a type II endoleak following endovascular repair of abdominal aortic aneurysm. Cardiovasc Intervent Radiol. 2003;26(5):482-4.

23. Baum RA, Carpenter JP, Cope C, et al. Aneurysm sac pressure measurements after endovascular repair of abdominal aortic aneurysms. J Vasc Surg. 2001;33(1):32-41.

24. Mansueto G, Cenzi D, Scuro A, et al. Treatment of type II endoleak with a transcatheter transcaval approach: results at 1-year follow-up. J Vasc Surg. 2007;45(6):1120-7.

25. Yang RY, Tan KT, Beecroft JR, Rajan DK, Jaskolka JD. Direct sac puncture versus transarterial embolization of type II endoleaks: an evaluation and comparison of outcomes. Vascular. 2017;25(3):227-33.

26. Chung R, Morgan RA. Type 2 endoleaks post-EVAR: current evidence for rupture risk, intervention and outcomes of treatment. Cardiovasc Intervent Radiol. 2015;38(3):507-22.

27. Baum RA, Stavropoulos SW, Fairman RM, Carpenter JP. Endoleaks after endovascular repair of abdominal aortic aneurysms. J Vasc Interv Radiol. 2003;14(9 Pt 1):1111-7.

28. Stavropoulos SW, Park J, Fairman R, Carpenter J. Type 2 endoleak embolization comparison: translumbar embolization versus modified transarterial embolization. J Vasc Interv Radiol. 2009;20(10):1299-302.
29. Kuziez MS, Sanchez LA, Zayed MA. Abdominal aortic aneurysm type II endoleaks. J Cardiovasc Dis Diagn. 2016;4(5):255.

30. Golzarian J, Maes EB, Sun S. Endoleak: treatment options. Tech Vasc Interv Radiol. 2005;8(1):41-9.

31. Muller-Wille R, Wohlgemuth WA, Heiss P, et al. Transarterial embolization of type II endoleaks after EVAR: the role of ethylene vinyl alcohol copolymer (Onyx). Cardiovasc Intervent Radiol. 2013;36(5):1288-95.

32. Khaja MS, Park AW, Swee W, et al. Treatment of type II endoleak using Onyx with long-term imaging follow-up. Cardiovasc Intervent Radiol. 2014;37(3):613-22.

33. Sidloff DA, Stather PW, Choke E, Bown MJ, Sayers RD. Type II endoleak after endovascular aneurysm repair. Br J Surg. 2013;100(10):1262-70.

34. Angle JF. Cone-beam CT: vascular applications. Tech Vasc Interv Radiol. 2013;16(3):144-9.

35. Binkert CA, Alencar H, Singh J, Baum RA. Translumbar type II endoleak repair using angiographic CT. J Vasc Interv Radiol. 2006;17(8):1349-53.

36. Choi SY, Won JY, Lee DY, et al. Percutaneous transabdominal approach for the treatment of endoleaks after endovascular repair of infrarenal abdominal aortic aneurysm. Korean J Radiol. 2010;11(1):107-14.

37. Rial R, Serrano Fj F, Vega M, et al. Treatment of type II endoleaks after endovascular repair of abdominal aortic aneurysms: translumbar puncture and injection of thrombin into the aneurysm sac. Eur J Vasc Endovasc Surg. 2004;27(3):333-5.

38. Molyneux AJ, Coley SC. Embolization of spinal cord arteriovenous malformations with an ethylene vinyl alcohol copolymer dissolved in dimethyl sulfoxide (Onyx liquid embolic system). Report of two cases. J Neurosurg. 2000;93(2 Suppl):304-8.

39. Scali ST, Vlada A, Chang CK, Beck AW. Transcaval embolization as an alternative technique for the treatment of type II endoleak after endovascular aortic aneurysm repair. J Vasc Surg. 2013;57(3):869-74. 